\title{
3D Printed Microneedle Array for Electroporation
}

\author{
Khalil Moussi, Member, IEEE, Mincho Kavaldzhiev, Jose E. Perez, Nouf Alsharif, \\ Jasmeen Merzaban, Jürgen Kosel, Senior Member, IEEE
}

\begin{abstract}
In-vitro transfection of cells by electroporation is a widely used approach in cell biology and medicine. The transfection method is highly dependent on the cell culture's electrical resistance, which is strongly determined by differences in the membranes, but also on the morphology of the electrodes. Microneedle (MN)-based electrodes have been used to concentrate the electrical field during electroporation, and therefore maximize its effect on cell membrane permeability. So far, the methods used for the fabrication of MN electrodes have been relatively limited with respect to the needle design. In this work, we provide a method to fabricate MNs using 3D printing, which is a technology that provides a high degree of flexibility with respect to geometry and dimensions. Pyramidal-shaped MN designs were fabricated and tested on HCT116 cancer cells. Customization of the tips of the pyramids permits tailoring of the electrical field in the vicinity of the cell membranes. The fabricated device enables low-voltage $(2 \mathrm{~V})$ electroporation, eliminating the need for the use of specialized chemical buffers. The results show the potential of this method, which can be exploited and optimized for many different applications, and offer a very accessible approach for in-vitro electroporation and cell studies. The MNs can be customized to create complex structures, for example, for a multi-culture cell environment.

Keywords-Electroporation, Microneedles, Transfection, 3D printing.
\end{abstract}

\section{INTRODUCTION}

Gaining intracellular access is of crucial importance in research areas such as cell biology, gene editing, and neurology. The main difficulty in developing methods for overcoming the cell membrane barrier is that the cells are morphologically heterogeneous even in mono cell cultures [1], [2]. Moreover, the direct cell membrane breaching is challenging due to its elasticity and conformability with nano and microobjects [3]. The techniques used for the delivery of molecules across the cell membrane are lipid-mediated [4], peptides [5], viral vectors [6], electroporation [7], active scaffolds [8]. Out of those, the most common method is electroporation. For electroporation, the transfection is achieved by the application of an electric potential higher than the transmembrane breakdown voltage. Thus, opening nanopores in the cell membrane and allowing molecules to enter into the cell cytosol. The transmembrane breakdown voltage has been reported to be 0.2-1 V [9], [10]. Traditionally, electroporation is performed in bulk by applying high electrical potentials

K. Moussi, M. Kavaldzhiev, and J. Kosel are with the Computer, Electrical and Mathematical Science and Engineering division at King Abdullah University of Science and Technology, Thuwal, Saudi Arabia.

J. E. Perez, N. Alsharif and J. Merzaban are with the Biological Environmental Science and Engineering division at King Abdullah University of Science and Technology, Thuwal, Saudi Arabia.

E-mail:\{khalil.moussi, jasmeen.merzaban, jurgen.kosel\}@ kaust.edu.sa. (typically $\mathrm{kV}$ ) between two metal plates porating a large number of cells at the same time [11], [12]. The application of high electrical potentials has drawbacks, e.g., to generate a high concentration of lipid oxide species in the cell membrane, ultimately lowering the cells' viability [13], [14]. Moreover, in conventional electroporation, the entire population is always affected. The lack of localized or controlled means of electroporation characterizes this design. In order to create opportunities for new applications and more complex studies, devices with complex designs will be required that allow, for example, localized electroporation within a cell culture.

Currently, to overcome oxidizing lipid effects and to customize the electroporation pulses for different cells, two approaches were reported: firstly, by shortening the electrical pulse duration [9], secondly, by modifying the electrodes [15], e.g., distance, surface morphology or shape. Suitable systems that incorporate these features are micro/nanoneedle arrays, which have been used for singlecell and multi-cell cultures [16]-[19]. The shape of the needles enhances the electrical fields in the vicinity of the cell membrane, allowing them to perform the electroporation at lower voltages (50-2.5 V), yet, with high transfection efficiency [19].

This study aims to achieve a customizable electroporation platform that provides freedom regarding the design and the electrode arrangement. These requirements can be achieved by taking advantage of the flexibility of additive manufacturing [20]. This method allows fabricating MNs and tailor their poration features. The complete electroporation device (Fig. 1) consists of a transparent indium tin oxide (ITO) electrode cover at the top and an electroporation chamber

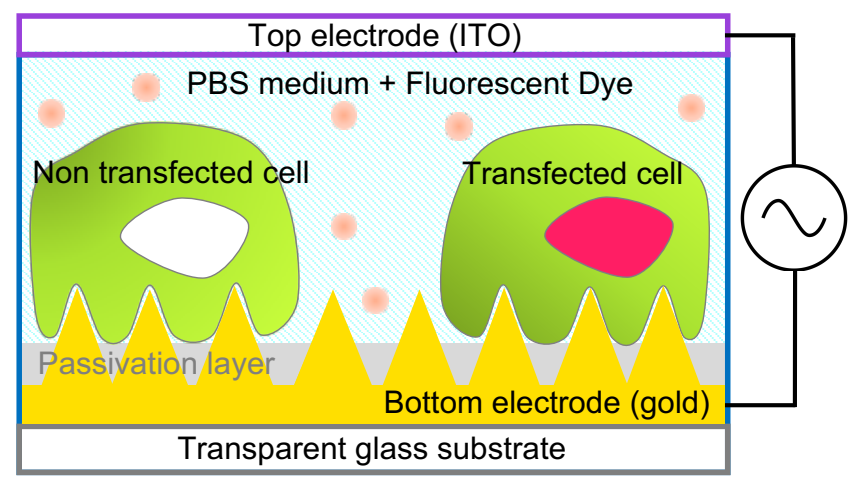

Fig. 1: Schematic diagram of the electroporation system and illustration of the operation. 
with 3D printed microelectrodes at the bottom. The bottom and top electrodes are separated by a $10 \mu \mathrm{m}$ thick SU-8 photoresist layer. A passivation layer was added to reduce the risk of electrolysis, promote cell contact with the MN tips, and improve cell attachment. HCT116 cells were growing on top of the MN array in phosphate-buffered saline (PBS) solution for $24 \mathrm{~h}$ after seeding. Double staining of cells with fluorescent dyes calcein-AM (green dye) and ethidium homodimer-1 (EthD-1) (red dye) was used to quantify intracellular access by electroporation. The green dye calcein-AM indicates live cells, while the red dye EthD-1 cannot access the cell cytoplasm unless the cell membrane is compromised (i.e., as in cell death). Therefore, the colocalization of the two dyes can only be expected if the cell is alive, and EthD-1 has been transported inside the cells via electroporation.

\section{METHODS}

\section{A. Fabrication}

The 3D printed pyramidal MNs were prepared using a direct laser writing system (Nanoscribe, GmbH, Photonics Professional GT) [21], utilizing two-photon polymerization of photoresist (IP-L780, Nanoscribe, $\mathrm{GmbH}$ ) that was drop cast on a borosilicate glass coverslip (area of $2.5 \mathrm{~cm}^{2}$ and thickness of $180 \mu \mathrm{m}$ ) (Fig. 2 (a-b)). The glass coverslip was mounted in dip-in laser lithography (DILL) configuration, and the laser output power was adjusted to $160 \mathrm{~mW}$ using the $63 x$ objective lens (Zeiss microscope objective NA 1.4). The development of the photoresist was carried out in a beaker, where the pyramidal MNs were positioned vertically, filled with $40 \mathrm{ml}$ of mr-DEV 600 developer (micro resist technology, $\mathrm{GmbH}$ ) for 3 minutes and 30 seconds, followed by dipping into isopropyl alcohol for 30 seconds and drying with nitrogen (Fig. 2 (c) and (f)). Next, a gold film (902 PLC, Equipment Support Company Ltd) was sputter deposited at room temperature onto the substrate with a thickness of 100 $\mathrm{nm}$ with $15 \mathrm{~nm}$ of titanium for adhesion. The pressure in the deposition chamber was maintained at $9 \times 10^{-7}$ Torr, and the deposition rates were $0.3 \mathrm{~nm} / \mathrm{s}$ for gold and titanium. The electroporation chamber was made following the subsequent steps, spin coating SU8 2010 photoresist (micro resist technology, $\mathrm{GmbH}$ ), pre-bake for 3 minutes at $95^{\circ} \mathrm{C}$, $\mathrm{UV}$ exposure through a mask with $120 \mathrm{~mJ} / \mathrm{cm}^{2}$, post-exposure bake of 3 minutes at $95^{\circ} \mathrm{C}$, dip in SU8 developer for 2 minutes, and clean with isopropyl alcohol for 20 seconds. The electroporation chamber covered an area of $1.44 \mathrm{~mm}^{2}$ (Fig. 2 (d)). Finally, an isolation layer of SU8 2000.5 photoresist was applied by spin coating. Then pre-baked for 1 minute followed by $90 \mathrm{~mJ} / \mathrm{cm}^{2}$ of exposure, $1 \mathrm{~min}$ of post-exposure bake with 30 seconds of development in SU8 developer (Fig. 2 (g) and (h)). The top electrode was made from a Borosilicate glass coverslip (180 $\mu \mathrm{m}$ thickness, Fisher- brand, 12-543A), which was coated on both sides with indium tin oxide (ITO) by sputter deposition (902 PLC, Equipment Support Company Ltd) (Fig. 2 (e)).

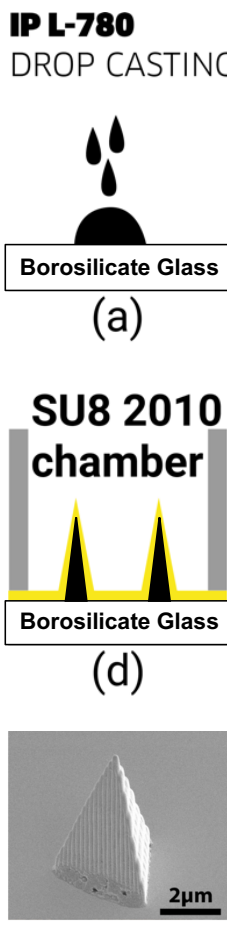

(f)

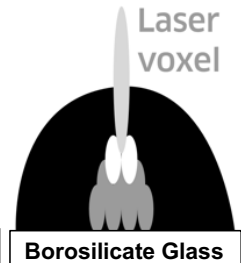

(b)

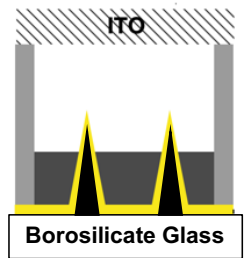

(e)

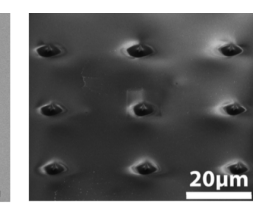

(g)

\section{Printed pyramids}

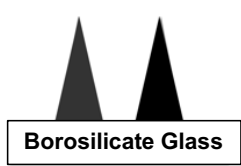

(c)

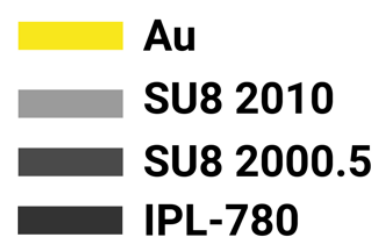

Fig. 2: Illustration of the fabrication process of the microneedle electroporation platform. (a) Drop casting of a photoresist on a borosilicate glass substrate, (b) Direct laser writing of the structures. (c) Developed pyramidal microneedles. (d) Electroporation chamber walls fabrication by spin coating and lithography of SU8 2010. (e) Passivation layer of SU8 2000.5 and closure of the device with a glass cover with sputtered ITO electrode. The gap between the microneedles tip and the ITO electrode is $5 \mu \mathrm{m}$. (f) Scanning electron microscope (SEM) image of a gold coated pyramidal microneedle with $6 \mu \mathrm{m}$ height, and $280 \mathrm{~nm}$ tip. (g) SEM image of a patch of needles with SU8 2000.5 photoresist as passivation layer in-between, showing uncovered microneedles tips. (h) Cross-section SEM image of a gold coated microneedle with SU8 2000.5 photoresist passivation.

\section{B. Cell line HCT116 preparation and handling}

HCT116 colorectal carcinoma cells (ATCC ${ }^{\circledR}$ CCL-247) were grown in McCoy's 5A modified medium (ATCC ${ }^{\circledR}$ ), supplemented with $10 \%$ fetal bovine serum (Gibco $\left.{ }^{\mathrm{R}}\right)$ and cultured at $37^{\circ} \mathrm{C}$ in a humidified incubator with $5 \% \mathrm{CO} 2$, as indicated by the vendor. After reaching the desired confluency, the cells were detached using Accutase (StemPro ${ }^{\circledR}$ ) and quantified via the trypan blue method. Before cell seeding, the MN substrate was first sterilized in $100 \%$ ethanol for an hour then thoroughly washed with PBS and cell medium. The cells were allowed to grow for 24 hours before all experiments were performed. The cytotoxicity of the MNs was evaluated using the LIVE/DEAD Viability/Cytotoxicity Kit (Molecular ProbesTM), following vendor indications. Briefly, the cells were washed with PBS and then stained with a $2 \mu \mathrm{M}$ calcein-AM / $4 \mu \mathrm{M}$ ethidium homodimer-1 (EthD-1) solution for 35 minutes at room temperature. The cells were then washed with PBS and imaged using a Leica DMI6000 B fluorescence microscope. 

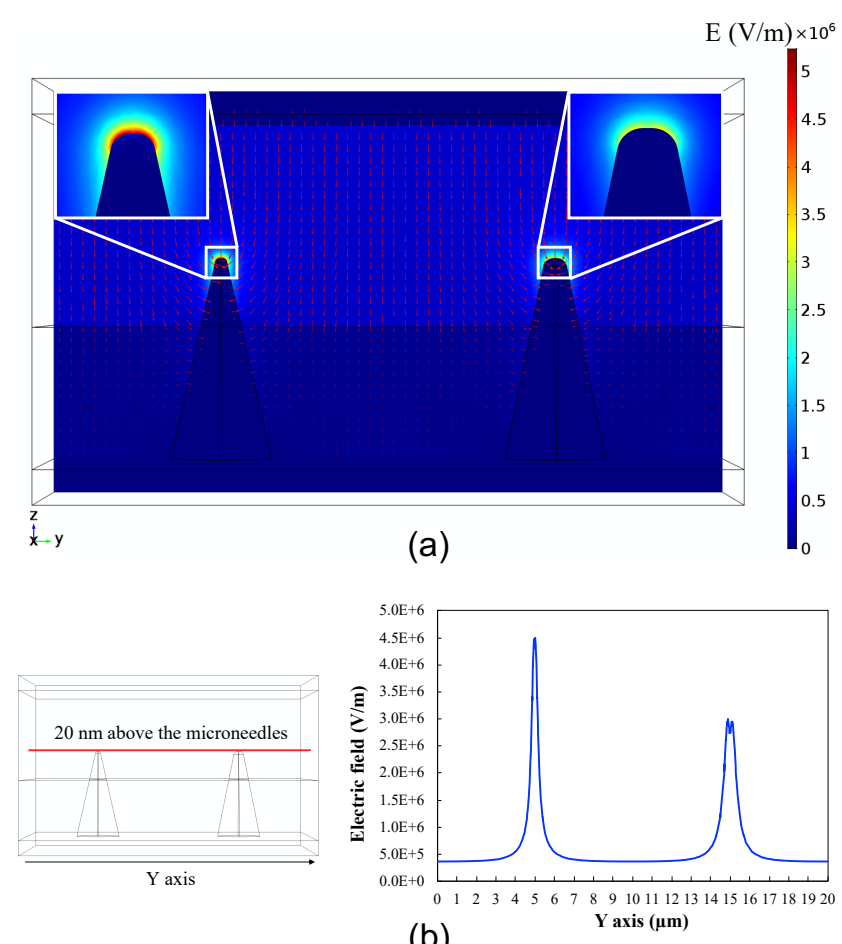

(b)
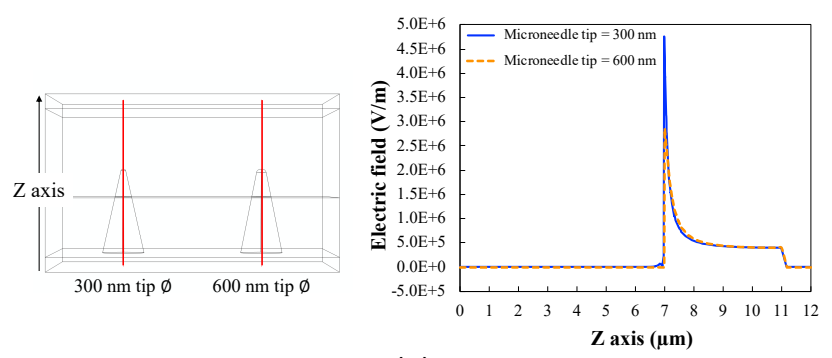

(c)

Fig. 3: FEM results. (a) Color plot of the electric field distribution between top and bottom electrodes for two microneedles with different tip diameters (300 $\mathrm{nm}$ and $600 \mathrm{~nm}$ ). (b) Electric field in proximity of the microneedles tips (20 $\mathrm{nm}$ above the microneedles). (c) Electric field along the $\mathrm{Z}$ axis (left image showing the measurement lines).

\section{Electrical setup}

The electroporation experiments were carried out by applying unipolar pulses with amplitude of $2 \mathrm{~V}$ and frequency of $100 \mathrm{~Hz}$ using a function generator (Agilent 33220A). The pulse width was $250 \mu \mathrm{s}$, and different numbers of pulses were applied.

\section{Finite ELEMENT MODELING}

The finite element method (FEM) was used to study the electric field distribution and the effect of different MN tip designs in terms of the resulting electrical fields. The FEM was implemented using COMSOL Multiphysics. The electrostatic 2D model consisted of two conducting planes with a thickness of $1 \mu \mathrm{m}$ from top and bottom, separated by a $10 \mu \mathrm{m}$ gap. In addition to two conic MNs on the bottom electrode, one with a tip and base diameters of $300 \mathrm{~nm}$ and $5 \mu \mathrm{m}$, respectively. The other, with a tip and base diameters of $600 \mathrm{~nm}$ and $5 \mu \mathrm{m}$, respectively. Both MNs were $6 \mu \mathrm{m}$ high. The boundary condition of the bottom electrode (plate and $\mathrm{MNs}$ ) was set to $0 \mathrm{~V}$ and the one of the top electrode was set to $2 \mathrm{~V}$. Similar, to the experimental setup, the MNs were isolated using a block with a permittivity of 3.75 (the permittivity of the SU 8 photoresist) and height of $5 \mu \mathrm{m}$.

The electric field is concentrated around the tips (Fig. 3(a)) with a value of about $4.5 \mathrm{MV} / \mathrm{m}$, while the electric field in a homogeneous space surrounding the MNs is ten times lower, around $0.5 \mathrm{MV} / \mathrm{m}$, confirming the substantial field enhancement achieved by the MN structures. This aids in reducing the applied voltage for the electroporation. The impact of the tip's diameter on the field distribution is shown in Figure 3 (a-c), where an increase of the diameter from 300 $\mathrm{nm}$ to $600 \mathrm{~nm}$ results in a field reduction of about $1.5 \mathrm{MV} / \mathrm{m}$ (30\%). Hence, by customizing the tip of the 3D MN, the electric field distribution can be tailored, and the versatility of 3D printing can be exploited to optimize the electroporation characteristics.

\section{RESULTS AND DISCUSSIONS}

The biocompatibility of the SU8 photoresist has been investigated following a viability assay of the HCT116 cell line. After incubation of the HCT116 cells for 24h on top of the SU8 2000.5 photoresist, they exhibit a regular spreading across the photoresist with no dead cells present (Fig. 4 (a)), confirming the high biocompatibility of the photoresist. The same biocompatibility assay was performed on top of the pyramidal MNs and showed excellent viability of the cells $24 \mathrm{~h}$ and $72 \mathrm{~h}$ after seeding (Fig. 4 (b, c)).

$24 \mathrm{~h}$ after seeding, the quantification of intracellular delivery by electroporation was based on the double staining of cells with calcein-AM and EthD-1. The calcein-AM/EthD-1 dye combination works through exclusion: while the calceinAM (green dye) passes through the cell membrane and stains live cells, the EthD-1 (red dye) cannot access the cell cytoplasm unless the cell membrane is compromised (i.e., as in cell death). Therefore, a double-stained cell with calcein and EthD-1 can only be expected if the cell is alive, and EthD-1 has been transported inside of the cells. In this case, we assume that the cell electroporation would affect the cell membrane in contact with the MNs, opening up a gap for a

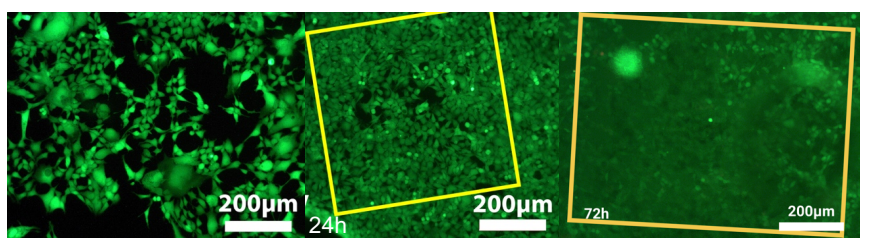

(a)

(b)

(c)

Fig. 4: Optical fluorescent microscopy images of cancel HCT116 cells stained with calcein AM (green) dye. (a) Cells grown on an SU8 2000.5 layer after $24 \mathrm{~h}$. (b) Cells grown on top of microneedles after $24 \mathrm{~h}$ and (c) after $72 \mathrm{~h}$. The yellow rectangles in (b) and (c) designate the pyramidal needle locations. 


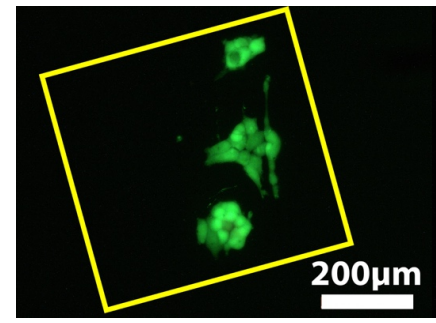

(a)

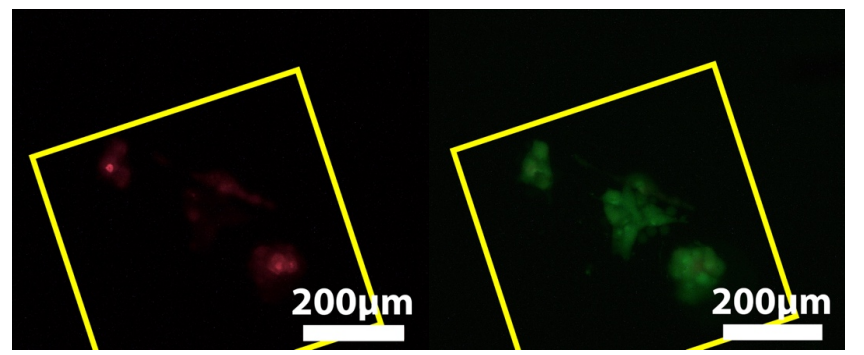

(c)

(d)
Fig. 5: Optical fluorescent microscopy of HCT116 cells stained with calcein (green) and EthD-1 (red) fluorescent dyes. (a) Cancer cells stained with calcein AM on top of microneedle array electrode. (b) Transfection of 25\% of the cells with a train of 500 pulses of $2 \mathrm{~V}$ and $250 \mu$ s width. (c) Transfection of $80 \%$ of the cells after application of additional 1000 pulses. (d) The viability of the cells after electroporation evaluated with calcein AM.

molecule to gain intracellular access without compromising cell viability. Direct quantification of the number of transfected cells yielded a delivery efficiency of $25 \%$, evidenced by the colocalization of the two cell viability dyes (Fig. $5(\mathrm{a}, \mathrm{b}))$.

Figure 5 (a) shows HCT116 cells grown on top of the MNs electrode $24 \mathrm{~h}$ after seeding. The green fluorescent dye (calcein) is indicating alive cells. After applying the first train of 500 pulses , delivery of the red fluorescent dye (EthD-1) was achieved, as observed in Fig. 5 (b).

By applying an additional train of 1000 pulses, a very high transfection of about $80 \%$ was achieved (Fig. 5 (c)). As can be seen from Fig. 5 (d), cell viability was maintained after applying the electroporation voltage pulses. The procedure of consecutive pulse trains with relaxation time preserved the chances of the solution to maintain the cells alive from overheating and other undesirable electrical effects (change of the buffer $\mathrm{pH}$ ).

\section{CONCLUSION}

In a step towards controllable and localized electroporation, a customizable platform based on 3D printed pyramidalshaped MNs has been fabricated. The fabrication method provides high flexibility in the design and electrode arrangement. Using FEM simulation, we demonstrated that changing the tip-curvature allows tailoring the electric field around the microneedles. Electroporation of HCT116 cancer cells grown on top of the MNs has been achieved by applying 1500 pulses with a voltage amplitude and pulse width of $2 \mathrm{~V}$ and $250 \mu \mathrm{s}$, respectively. The quantification of transfected cells after delivery of the red fluorescent dye (EthD-1) yielded a delivery efficiency of about $80 \%$ and high viability.

\section{REFERENCES}

[1] S. J. Altschuler and L. F. Wu. "Cellular heterogeneity: do differences make a difference?," Cell, vol. 141, no. 4, pp. 559-563, 2010.

[2] B. Snijder and L. Pelkmans, "Origins of regulated cell-to-cell variability," Nature reviews Molecular cell biology, vol. 12, no. 2, p. 119-125, 2011.

[3] L. Hanson, Z. C. Lin, C. Xie, Y. Cui, and B. Cui, "Characterization of the Cell-Nanopillar Interface by Transmission Electron Microscopy," Nano Lett, vol. 12, no. 11, pp. 5815-5820, 2012.

[4] B. Küçüktürkmen and A. Bozkır, "Development and characterization of cationic solid lipid nanoparticles for co-delivery of pemetrexed and miR-21 antisense oligonucleotide to glioblastoma cells," Drug Dev and Ind Pharm, vol. 44, no. 2, pp. 306-315, 2018.

[5] M. Gagat, Z. Wioletta, and A. Grzanka, "Cell-penetrating peptides and their utility in genome function modifications," International journal of molecular medicine, vol. 40, no. 6, pp. 1615-1623, 2017.

[6] C. Geisler and D.L. Jarvis, "Adventitious viruses in insect cell lines used for recombinant protein expression," Protein Expression Purification, vol. 144, pp. 25-32, 2018.

[7] D. Zhao, D. Huang D, Y. Li, M. Wu, W. Zhong, et al., "A FlowThrough Cell Electroporation Device for Rapidly and Efficiently Transfecting Massive Amounts of Cells in vitro and ex vivo," Sci Rep., vol. 6, no. 1, pp. 1-9, 2016.

[8] X. Zhao, J. Kim, C. A. Cezar, N. Huebsch, K. Lee, K. Bouhadir, et al., "Active scaffolds for on-demand drug and cell delivery," Proc Natl Acad Sci., vol. 108, no. 1, pp. 67-72, 2011.

[9] J. Gehl, "Electroporation: theory and methods, perspectives for drug delivery, gene therapy and research," Acta Physiologica Scandinavica, vol. 177, no. 4, pp. 437-447, 2003.

[10] I. van Uitert, S. Le Gac, and A. van den Berg, "The influence of different membrane components on the electrical stability of bilayer lipid membranes," Biochimica et Biophysica Acta (BBA)-Biomembranes, vol. 1798, no. 1, pp. 21-31, 2010.

[11] C. E. Suarez and T. F. McElwain, "Transient transfection of purified Babesia bovis merozoites," Experimental parasitology, vol. 118 no. 4, pp. 498-504, 2008.

[12] M. Soueid, M. C. F. Dobbelaar, S. Bentouati, S. M. Bardet, R. P. O'Connor, et al., "Delivery devices for exposure of biological cells to nanosecond pulsed electric fields," Medical \& biological engineering \& computing, vol. 56, pp. 85-97, 2018.

[13] M. Breton and L. M. Mir, "Investigation of the chemical mechanisms involved in the electropulsation of membranes at the molecular level," Bioelectrochemistry, vol. 119, pp. 76-83, 2018.

[14] M. Yusupov M, J. Van der Paal, E. C. Neyts, and A. Bogaerts, "Synergistic effect of electric field and lipid oxidation on the permeability of cell membranes," Biochimica et Biophysica Acta (BBA)-General Subjects, vol. 1861, no. 4, pp. 839-847, 2017.

[15] J. Experton, A. G. Wilson and C. R. Martin, "Low-Voltage FlowThrough Electroporation in Gold-Microtube Membranes," Analytical chemistry, vol. 88, no. 24, pp. 12445-12452, 2016.

[16] S. O. Choi, Y. C. Kim, J. H. Park, J. Hutcheson, H. S. Gill, et al., "An electrically active microneedle array for electroporation," Biomedical Microdevices, vol. 12, no. 2, pp. 263-273, 2010.

[17] C. Xie, Z. Lin, L. Hanson, Y. Cui, and B. Cui, "Intracellular recording of action potentials by nanopillar electroporation," Nature Nanotechnology, vol. 7 no. 3, pp. 185-190, 2012.

[18] Y. Zu, S. Huang, Y. Lu, X. Liu, and S. Wang, "Size specific transfection to mammalian cells by micropillar array electroporation," Scientific reports, vol. 7, no. 6, pp. 38661, 2016.

[19] X. Xie, A. M. Xu, S. Leal-Ortiz, Y. Cao, C. C. Garner, and N. A. Melosh, "Nanostraw-Electroporation System for Highly Efficient Intracellular Delivery and Transfection," ACS Nano, vol. 7, no. 5, pp. 4351-4358, 2013.

[20] K. Moussi, A. Bukhamsin, T. Hidalgo, and J. Kosel, "Biocompatible 3D Printed Microneedles for Transdermal, Intradermal, and Percutaneous Applications," Advanced Engineering Materials, pp. 1901358, 2019.

[21] K. Moussi and J. Kosel, "3-D Printed Biocompatible Micro-Bellows Membranes," J. Microelectromech. Syst., vol. 27, no. 3, pp. 472-478, 2018. 